oder

$$
w_{i}=\frac{\alpha}{\delta t \cdot \varepsilon \sqrt{2}} \delta_{i} H
$$

wo $\delta_{i} H$ der Zunahme des Integrals $H$ bei der Vergrösserung des Arguments $t$ von $t_{i}$ auf $t_{i}+\delta t$ entspricht. Hierbei sind $t_{i}$ und $t_{i}+\delta t$ zwei in der Tabelle auf einander folgende Werthe dieses Argumentes, zwischen welchen der Werth $\tau_{i} \alpha: \varepsilon \sqrt{2}$ liegt.

In ähnlicher Weise ist

$$
W_{i}=\frac{\alpha}{\delta t \cdot \varepsilon \sqrt{2}} \Delta_{i} H
$$

und folglich

$$
\frac{w}{W}=\frac{\| \delta_{i}}{\| \Delta_{i}}
$$

Für unser Beispiel erhält man nach dieser Formel

$$
\frac{w}{W}=0.784
$$

wenn man $\delta_{i}$ und $\Delta_{i}$ aus den Burgess'schen Tabellen des Integrals $H$ für $\delta t=0.00 \mathrm{I}$ entnimmt.

13. Doch kann man, wenn man die Formel (16) benutzen will, eine directe Bestimmung der wahrscheinlichsten Fehlergesammtheit, die immer mühsam ist, entbehren, weil für uns nicht diese Gesammtheit selbst, sondern nur das derselben entsprechende Product $\Pi \Delta_{i}$ von Wichtigkeit ist.

Da die Werthe der Wurzeln $b_{i}: \varepsilon \sqrt{2}$ der Gleichungen (8) ausschliesslich von dem Werthe $N$ abhängen, ist es klar, dass auch die Grössen $\tau_{i} \alpha: \varepsilon \sqrt{\mathbf{2}}$, mit denen diese Wurzel vermittelst der Gleichungen (9) verbunden sind, bei genügend kleinem $\alpha: \varepsilon$ fast ausschliesslich von $N$ abhängen.

Unter solcher Voraussetzung entspricht jedem Werthe von $N$ eine bestimmte oder, genauer gesagt, eine sehr wenig mit den Aenderungen von $\alpha$ und $\varepsilon$ veränderliche Gesammtheit der Werthe von $\Delta_{i}$. Deswegen kann man näherungsweise

$$
\Pi \Delta_{i}=\varphi(N)=\Delta^{N}
$$

annehmen, wo $\Delta$ eine Function ausschliesslich von $N$ sein wird; das wird der geometrische Mittelwerth der Tabellendifferenzen, welche dem Werthe des Integrals $H$ nach $N$ aequidistanten Werthen des Argumentes $t$ entsprechen.
Die Werthe von $\Delta$ habe ich für verschiedene $N$ nach den Tabellen von Burgess, wo $\delta t=0.001$ ist, und nach den Tabellen, die bei Chauvenet (Astronomy, 2, p. 593) gegeben sind und wo $\delta t=0.01$ ist, berechnet. Die Logarithmen derselben sind in folgender Tabelle gegeben

\begin{tabular}{r||c|c||c|c}
\hline \multicolumn{1}{c||}{$N$} & \multicolumn{2}{c||}{ Burgess } & \multicolumn{2}{c}{ Chauvenet } \\
& $\lg \Delta_{\mathrm{B}}$ & $\lg \Delta_{\text {ber. }}$ & $\lg \Delta_{\mathrm{C}}$ & $\lg \Delta_{\text {ber. }}$ \\
\hline \hline \multirow{2}{*}{10} & 7.84814 & 7.84832 & 8.84829 & 8.84832 \\
20 & 7.84212 & 7.84207 & 8.84257 & 8.84207 \\
25 & 7.84075 & 7.84070 & 8.84070 & 8.84070 \\
50 & 7.83809 & 7.83761 & 8.83789 & 8.83761 \\
100 & 7.83553 & 7.83635 & 8.83682 & 8.83635
\end{tabular}

und können durch eine empirische Formel

$$
\lg \Delta=\lg \delta t+9.83482+\frac{0.155}{N}=\frac{0.2}{N^{2}}
$$

verbunden werden. Diese Formel stellt die unmittelbar berechneten Werthe ziemlich gut dar, wie man aus der Vergleichung derselben mit den oben gegebenen Werthen $\lg \Delta_{\text {ber. }}$ sehen kann.

Für das Verhältniss der Wahrscheinlichkeiten $w$ und $W$ bekommen wir also endlich folgende Formel

$$
\frac{\mathfrak{U}}{W}=\frac{\boldsymbol{U} \delta_{i}}{\Delta^{N}}
$$

Für unsèr Beispiel ist nach der Formel (19)

$$
\frac{w}{W}=0.731 \text {. }
$$

14. Praktisch können also alle Berechnungen auf folgende vier Operationen beschränkt werden :

$\left.\mathbf{I}^{\circ}\right)$ Die Bestimmung der mittleren quadratischen Fehler $\varepsilon$ und die Darstellung aller Fehler als Functionen von $\varepsilon \sqrt{2}$.

$2^{\circ}$ ) Die Auffindung der Werthe der Tabellendifferenzen $\delta_{i}$ mit diesen Argumenten.

$3^{\circ}$ ) Die Berechnung der Grösse $\Delta$ nach der Formel (I 8).

$\left.4^{\circ}\right)$ Die Berechnung des Werthes des Verhältnisses $w: W$ nach der Formel (19).

Odessa, 1900 April.

Boris Weinberg.

\title{
Observations de planètes
}

avec l'équatorial de 14 pouces de l'observatoire de Bordeaux.

\begin{tabular}{l|l|l|l|l|l|l|l|l|}
\hline 1900 & T.m.Bord. & $\Delta$ AR. & $\Delta$ DP. & Cp. $\mid$ Obs. & AR.app. & $\log p . \Delta \mid$ & DP. app. $|\log p . \Delta|$ & Red. ad 1. app. $\mid *$ \\
\hline
\end{tabular}

1900 FG.

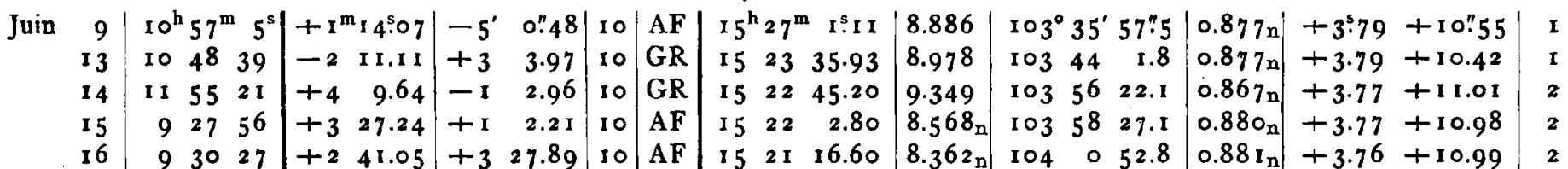




\begin{tabular}{|c|c|c|c|c|c|c|c|c|c|c|c|}
\hline 1900 & T.m.Bord. & $\triangle \mathrm{AR}$. & $\Delta \mathrm{DP}$. & $\mathrm{Cp}$. & Obs. & AR. app. & $\log p \cdot \Delta$ & DP. app. & $\log p . \Delta$ & Red. ad 1. app. & $*$ \\
\hline in 26 & $\operatorname{II}^{\mathrm{h}}{ }^{\mathrm{I}} 3^{\mathrm{m}} 34^{\mathrm{s}}$ & $+I^{m} 26:_{0}$ & $-I^{\prime} 27: 83$ & 10 & GR & $15^{\mathrm{h}} 14^{\mathrm{m}} 55^{\mathrm{s}} \mathrm{IO}$ & 9.390 & $104^{\circ} 30^{\prime} 23^{\prime \prime} 4$ & $0.868_{n}$ & $+3 \cdot 73+111^{5} \cdot 14$ & \\
\hline 28 & 94944 & +325.04 & $-3 \times 7.23$ & IO & AF & I 5 I $3 \quad 59.85$ & 9.045 & $1043^{6} \quad 49.9$ & & $71+11.30$ & 4 \\
\hline 30 & $10 \quad 32$ & +234.46 & 6.44 & Io & GR & $\begin{array}{lll}15 & 13 & 9.26\end{array}$ & $9 \cdot 314$ & $10444 \quad 13.5$ & $0.867 \mathrm{n}$ & $+3.70+11.25$ & 4 \\
\hline iill. & $95^{6}$ I 7 & +310.04 & to 54.48 & 10 & GR & I5 I2 47.40 & 9.182 & $104 \quad 47 \quad 57 \cdot 3$ & $0.879 n$ & $+3.69+11.33$ & \\
\hline 6 & $10 \quad 325$ & +129.07 & +2 I. $4 \mathrm{I}$ & 10 & AF & 151121.04 & 9.307 & 821.1 & $0.8 \eta 6_{n}$ & $+3.72+11.04$ & 6 \\
\hline 10 & $\begin{array}{lll}10 & 17 & 51\end{array}$ & +357.73 & $+53^{6.43}$ & 10 & $A F$ & $1510 \quad 52.86$ & 9.399 & $10525 \quad 54.5$ & $0.870_{n}$ & $+3.62+11.32$ & 7 \\
\hline
\end{tabular}

$1900 \mathrm{FH}$.

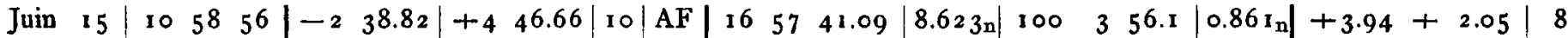

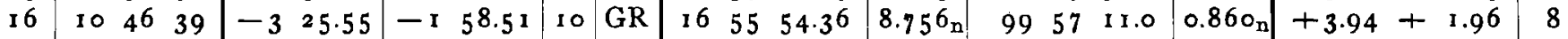

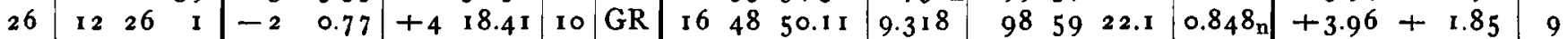
\begin{tabular}{l|lll|lll|lll|l|l|llll|l}
28 & 11 & 38 & 14 & -3 & 10.55 & -4 & 35.36 & I0 & GR & 16 & 47 & 40.33 & 9.152
\end{tabular}

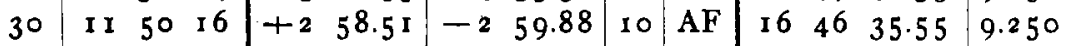

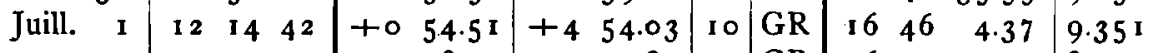
\begin{tabular}{llll|ll|ll|l|l|lll|l|}
7 & 10 & 27 & 7 & +3 & 8.12 & +2 & 54.82 & I0 & GR & I 6 & 43 & 35.70 & $8.924 \mathrm{n}$
\end{tabular}

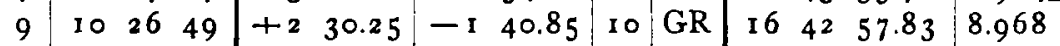

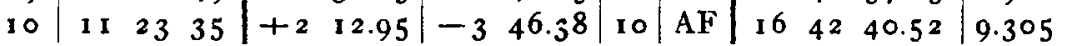
$985028.20 .85^{1} \mathrm{n}+3.96+1.719$ $984212.50 .848 n+3.94+2.1310$ $983819.20 .844 n+3.94+1.88$ I I $98201.20 .85^{1 n}+3.92+1.8612$ $981525.30 .849 n+3.92+1.7212$ Les observateurs sont: $\mathrm{AF}=\mathrm{M}$. A. Féraud, GR $=$ M. G. Rayet.

Positions moyennes des étoiles de comparaison.

\begin{tabular}{|c|c|c|c|}
\hline * & AR. I900.0 & DP. 1900.0 & Autorité \\
\hline I & $15^{\mathrm{h}} 25^{\mathrm{m}} 43^{\mathrm{s}} \cdot 25$ & $103^{\circ} 40^{\prime} 47^{\prime \prime} 4$ & $W_{1} 15.423$ \\
\hline 2 & $\begin{array}{lll}15 & 18 & 31.79\end{array}$ & $10357 \quad 13.9$ & $W_{1}$ I $5^{\mathrm{h}} \cdot 28 \mathrm{I}$ \\
\hline 3 & $15 \quad 13 \quad 25 \cdot 34$ & 1043140.1 & $W_{1}: 5^{h}: 183$ \\
\hline 4 & 151031.10 & 1043955.8 & $W_{1} 15^{h} \times 28, M_{1} 11108$ \\
\hline 5 & $15 \quad 9 \quad 33.67$ & $10446 \quad 51.5$ & $W_{1} 15^{h} I 11, A G$. Ott. \\
\hline 6 & 159988.25 & $105 \quad 6 \quad 6.6$ & $W_{1}=5^{\mathrm{h}} \times 15$ \\
\hline 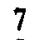 & I $5 \quad 6 \quad 51.49$ & $10520 \quad 6.7$ & $\mathrm{AOe}_{2}$ II $73^{1}$ \\
\hline 8 & $16 \quad 59 \quad 15.97$ & $99 \quad 59$ & $\begin{array}{l}\text { SD. }-9: 4483 \text {, rapp. à SD. } \\
-9.4510 \text { (AG.Ott.Z. I } 41)\end{array}$ \\
\hline
\end{tabular}

\begin{tabular}{r|rr|rr|r|r|}
\hline$*$ & AR. 1 900.0 & DP. 1900.0 \\
\hline 9 & $16^{\mathrm{h}}$ & $50^{\mathrm{m}} 46^{\mathrm{s}} .92$ & $98^{\circ}$ & $55^{\circ}$ & 1.8 & 5 \\
10 & 16 & 42 & 33.10 & 98 & 45 & 10.3 \\
111 & 16 & 42 & 5.94 & 98 & 33 & 23.2 \\
12 & 16 & 40 & 23.66 & 98 & 17 & 4.5 \\
\hline
\end{tabular}

Bordeaux, I 900 Juillet I I.

\section{Filar-Micrometer Observations of Asteroids}

made with the ra inch Equatorial of the Detroit Observatory, Ann Arbor, Mich., by Sidney $D$. Tozenley.

\begin{tabular}{|c|c|c|c|c|c|c|c|c|c|}
\hline 1897 & M.T.Ann Ar. & $\Delta \alpha$ & $\Delta \delta$ & $\mathrm{Cp}$ & $\alpha$ app. & $\log p \cdot \Delta$ & $\delta$ app. & $\log p .4$ & Red.ad l. app. \\
\hline
\end{tabular}

(137) Meliboea.

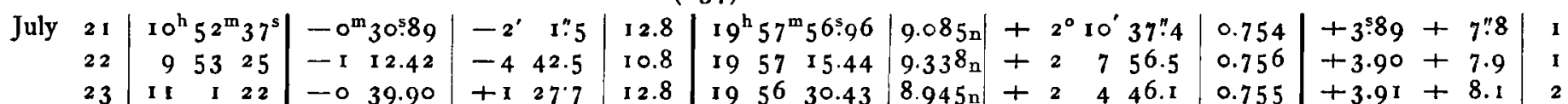

(200) Dynamene.

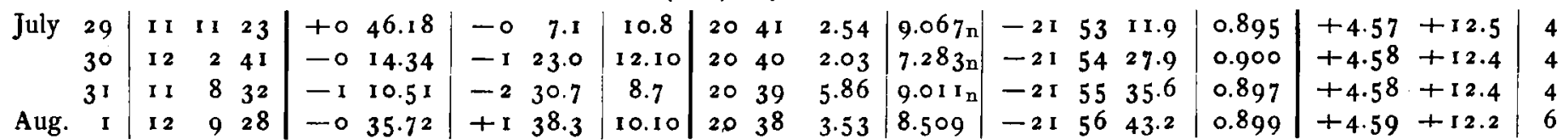

(79) Eurynome.

\begin{tabular}{|c|c|c|c|c|c|c|c|c|c|c|c|c|c|c|c|c|c|}
\hline July 30 & & & 38 & +0 & 53.8 & - & 4. & 10.8 & 20 & 24 & $3^{2 \cdot} \cdot 3^{2}$ & 43 & $-I I$ & 17 & 34 & 0.847 & $+4.28+10.9$ \\
\hline g. & & 6 & 35 & - & .74 & $+c$ & 11.2 & 10.10 & 20 & 22 & & 25 & -11 & 25 & . & & $+4.29+10.9$ \\
\hline 6 & & $3^{\circ}$ & 13 & -1 & I I & + & 9.9 & 10.10 & 20 & 17 & 5 & 8.500 & - I I & 45 & 3 I. 2 & 0.853 & $+4.33+10.9$ \\
\hline
\end{tabular}

